\title{
Sub-Synchronous Resonance Damper Based on the Stator Voltage Feedback for DFIG Wind Turbines
}

\author{
Javier Samanes, Leyre Rosado, Eugenio Gubia and Jesus Lopez \\ Electrical, Electronic and Communication Engineering \\ Institute of Smart Cities \\ Public University of Navarre (UPNA) \\ Pamplona, Spain \\ javier.samanes@unavarra.es
}

\begin{abstract}
Doubly-fed induction generator (DFIG) wind turbines connected to series compensated grids are prone to subsynchronous resonance (SSR) instability. In this paper we develop a model to analyze SSRs and propose a damping strategy based on the stator voltage feedback that is implemented in the rotorside converter (RSC). The control strategy is based on local variables that are already measured, so it is applicable to any new or existing DFIG wind turbine. Simulation results performed for a real wind farm where sub-synchronous resonances were reported validate the proposed damping strategy.
\end{abstract}

Index Terms-Doubly-fed Induction Generator (DFIG), SubSynchronous Resonance (SSR), wind turbine, resonance damping, frequency response

\section{INTRODUCTION}

The rapid growth of wind power is forcing to install wind farms far from the consumption centers, generally transporting the power generated through the existing transmission lines. In some cases, series capacitor compensation is required to avoid the additional cost of building new lines. However, the line series compensation interacts with DFIG wind turbines, creating oscillation problems that can lead to instability [1]. This phenomenon, in which the grid, the DFIG and the power converters and their control loops are involved, is referred as sub-synchronous resonances, and problems have been reported in Texas, Minnesota and China [2].

To avoid SSRs several damping strategies have been proposed for Flexible Alternating Current Transmission Systems (FACTS) [3] and for the two power converters used in DFIG turbines. The latest being the preferred option, as it does not require additional power converters.

The control loops of both the grid-side converter (GSC) and the RSC can be modified in order to damp SSRs and avoid instability. Some authors have proposed SSR dampers for the GSC [4], [5], others for the RSC [2], [6]-[8] while some others have combined damping strategies for both the RSC and the GSC [9], [10]. In the existing SSR damping strategies, the main variables used to damp the system are the voltage across the line series capacitor, the stator and rotor currents and the active power.

This work was supported by the Spanish State Research Agency (AEI) under grants PID2019-110956RB-I00/AEI/ 10.13039 and DPI-2016-80641R.
The voltage across the line series capacitor has been used to design effective SSR dampers to be used in the rotor or the grid power converters [4], [5], [7], [9]. This voltage is a remote signal, so it is normally estimated to avoid long communication lines. Other authors proposed the use of the stator and rotor currents, as they contain the sub-synchronous resonant components [2]. A different approach to damp SSRs is based on using the rotor currents to emulate a virtual resistor in series with the stator winding and a virtual inductance in series with the rotor winding [8]. The estimated series capacitor voltage can be combined with a virtual inductance implemented through the rotor current derivative [9]. SSRs can also be damped by notch filters inserted in the DC-voltage control loop of the GSC or in the inner current control loop of the RSC [10]. At last, a different variable such as the active power multiplied by a gain and a phase compensation can also damp the resonance [6].

Despite the variety of damping controllers, there is a really effective local variable that has not been considered yet, the stator voltage, or equivalently the output harmonic filter capacitor voltage. In this work, the damping capabilities of the stator voltage are explored and a robust damping controller is designed that can be used in any DFIG based wind farm, regardless of the line impedance compensation level and the wind turbine operating point.

\section{System Modeling and Stability Analysis}

\section{A. System Description and Model}

Fig. 1 illustrates a DFIG wind turbine connected to a series compensated grid. The RSC is directly connected to the rotor and controls the torque and rotational speed. The GSC controls the DC-bus, $C_{D C}$, voltage and is connected to the stator terminals through the converter inductor, $L_{G S C}$. The GSC output current, $i_{G}$, and the DFIG output current $i_{S}$ are filtered by an $L C L$ filter, formed by the capacitor $C_{f}$ and the wind turbine's step-up transformer, normally located inside the nacelle. This transformer has a leakage inductance $L_{t}$. The wind turbine is connected in parallel to the rest of turbines and to the grid at the point of common coupling (PCC). The grid is modeled as an ideal voltage source and a series impedance, formed by an inductor, $L_{g}$, and the series compensation capacitor, $C_{g}$. 


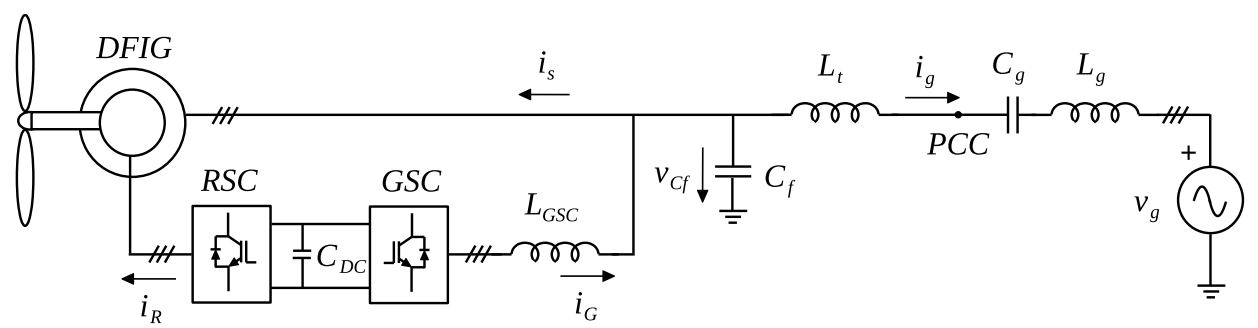

Fig. 1. DFIG wind turbine with an $L C L$ filter connected to a series compensated grid.

DFIG wind turbines are commonly controlled in the synchronous reference frame or $d q$ axis, so all the elements are modeled in this reference frame to properly study the system stability. In the following, $2 \times 2$ transfer function matrices and impedance matrices are used to model the system dynamics. This is a straightforward approach to model the cross-coupling terms between both axis and can be used for symmetric and non-symmetric systems [11], [12], the later being the case of the system under study when the phase-locked loop model, PLL, used to synchronize the power converter to the grid is included. The matrices obtained in the modeling procedure are denoted by capital letters between brackets, with a subscript that indicates the angular speed of the cross-coupling terms. Vectors are represented by capital letters, and the subscript $d q$ is used to denote that they are expressed in the synchronous reference frame.

Independent models are obtained for all the system components, which are later combined to obtain the complete plant model. This model considers the coupled dynamics between the GSC and the RSC, as they are both connected to the same terminals and both control loops interact with each other.

1) DFIG Model: The dynamics of a doubly-fed induction generator in $d q$ can be described by two equations

$$
V_{S d q}=\left[Z_{R L_{l S}}\right]_{\omega_{0}} I_{S d q}+\left[Z_{L_{m}}\right]_{\omega_{0}} I_{m d q}
$$

and

$$
V_{R d q}=\left[Z_{R L_{l R}}\right]_{\omega_{R}} I_{R d q}+\left[Z_{L_{m}}\right]_{\omega_{R}} I_{m d q},
$$

where the current, $I$, and voltage, $V$, subscripts, $R$ and $S$, stand for the rotor and stator, respectively. $I_{m d q}$ is the magnetizing current in $d q$ axis. $\left[Z_{R L_{l S}}\right]_{\omega_{0}}$ and $\left[Z_{R L_{l R}}\right]_{\omega_{R}}$ model the rotor and stator winding series resistance and the leakage inductance. $\omega_{0}$ is the rotational speed of the $d q$ axis, provided by the PLL, while $\omega_{R}$ is the difference between $\omega_{0}$ and the mechanical rotational speed $\omega_{m}$. The general expression for $\left[Z_{R L_{l}}\right]_{\omega}$ is

$$
\left[Z_{R L_{l}}\right]_{\omega}=\left[\begin{array}{cc}
R+L_{l} s & -L_{l} \omega \\
L_{l} \omega & R+L_{l} s
\end{array}\right],
$$

where $R=R_{S}, L_{l}=L_{l S}$ and $\omega=\omega_{0}$ for $\left[Z_{R L_{l S}}\right]_{\omega_{0}}$, while $R=R_{R}, L_{l}=L_{l R}$ and $\omega=\omega_{R}$ for $\left[Z_{R L_{l R}}\right]_{\omega_{R}}$. The last impedance, $\left[Z_{L_{m}}\right]_{\omega}$, models the magnetizing inductance

$$
\left[Z_{L_{m}}\right]_{\omega}=\left[\begin{array}{cc}
L_{m} s & -L_{m} \omega \\
L_{m} \omega & L_{m} s
\end{array}\right]
$$

Note that in (1) and (2) the impedance $\left[Z_{L_{m}}\right]_{\omega}$ is expressed at two different rotational speeds. To obtain an equivalent circuit for the DFIG in terms of impedance matrices, $\left[Z_{L_{m}}\right]_{\omega}$ has to be expressed in both equations at the same rotational speed. For this reason, a transformation matrix $[B]$ is proposed in this paper

$$
[B]=\left[\begin{array}{cc}
1+\frac{\omega_{R} \omega_{m}}{s^{2}+\omega_{R}^{2}} & -\frac{s \omega_{m}}{s^{2}+\omega_{R}^{2}} \\
\frac{s \omega_{m}}{s^{2}+\omega_{R}^{2}} & 1+\frac{\omega_{R} \omega_{m}}{s^{2}+\omega_{R}^{2}}
\end{array}\right],
$$

that multiplies both sides of (2). Doing so,

$$
[B] V_{R d q}=\left([B]\left[R_{R}\right]+\left[Z_{L_{l R}}\right]_{\omega_{0}}\right) I_{R d q}+\left[Z_{L_{m}}\right]_{\omega_{0}} I_{m d q} .
$$

It should be noticed that $\left[Z_{R L_{l R}}\right]_{\omega_{R}}$ in (2) is divided in (6) into the rotor winding series resistance $\left[R_{R}\right]$, to which the transformation matrix $[B]$ is applied, and the winding leakage inductance, $\left[Z_{L_{l R}}\right]_{\omega_{0}}$, with cross-coupling terms at $\omega_{0}$. (1) and (6) are the equations of the physical circuit represented in Fig. 2. These two equations can be rearranged, knowing that $I_{R d q}+I_{S d q}=I_{m d q}$, and expressed in terms of admittance matrices that capture the current dynamics as a function of the two model inputs, the rotor and stator voltages

$$
I_{S d q}=\left[Y_{I_{S} V_{R}}\right] V_{R d q}+\left[Y_{I_{S} V_{S}}\right] V_{S d q},
$$

and

$$
I_{R d q}=\left[Y_{I_{R} V_{R}}\right] V_{R d q}+\left[Y_{I_{R} V_{S}}\right] V_{S d q}
$$

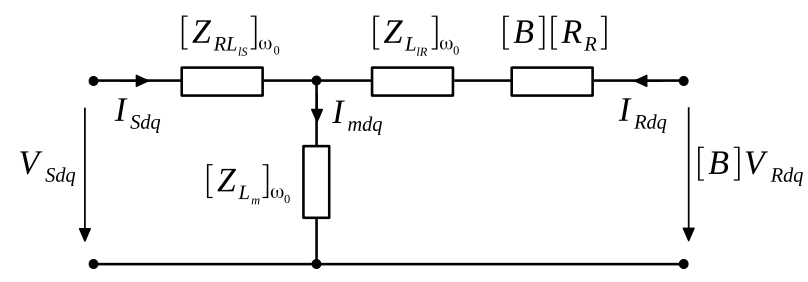

Fig. 2. DFIG equivalent circuit. 
2) Harmonic LC filter: The GSC output current, $I_{G d q}$, depends on the difference between the converter output voltage, $V_{G d q}$, and the filter capacitor voltage, $V_{C f d q}$

$$
I_{G d q}=\left[Z_{R L_{G}}\right]_{\omega_{0}}^{-1}\left(V_{G d q}-V_{C f d q}\right) .
$$

$\left[Z_{R L_{G}}\right]_{\omega_{0}}$ is a matrix impedance similar to (3) that models the GSC inductor, $L_{G S C}$, with a series resistor $R_{G S C}$. The filter capacitor, $C_{f}$, has a series damping resistor, $R_{d}$, and its impedance, $\left[Z_{C f}\right]_{\omega_{0}}$, is given by

$$
\left[Z_{C f}\right]_{\omega_{0}}=\left[\begin{array}{cc}
R_{d}+\frac{s}{C_{f}\left(s^{2}+\omega_{0}^{2}\right)} & \frac{\omega_{0}}{C_{f}\left(s^{2}+\omega_{0}^{2}\right)} \\
-\frac{\omega_{0}}{C_{f}\left(s^{2}+\omega_{0}^{2}\right)} & R_{d}+\frac{s}{C_{f}\left(s^{2}+\omega_{0}^{2}\right)}
\end{array}\right],
$$

so

$$
V_{C f d q}=\left[Z_{C f}\right]_{\omega_{0}} I_{C f d q} .
$$

From Fig. 1 it also becomes evident that the DFIG stator voltage and the filter capacitor voltage are equal,

$$
V_{C f d q}=V_{S d q} \text {. }
$$

3) Grid Model: The grid is modeled as a lumped inductance, $L_{g t}$. It includes the step-up transformer leakage inductance, $L_{t}$, and the distribution and wind farm grid inductance, as well as the wind farm substation, under the parameter $L_{g}$ of Fig.1. This inductance has a series resistance that models the resistance at $50 \mathrm{~Hz}$ of the elements lumped in $L_{g t} . C_{g}$ is the line series compensation capacitor. The grid impedance matrix, $\left[Z_{g}\right]_{\omega_{0}}$, is thus defined as

$\left[Z_{g}\right]_{\omega_{0}}=\left[\begin{array}{cc}R_{g}+L_{g t} s+\frac{s}{C_{g}\left(s^{2}+\omega_{0}^{2}\right)} & -L_{g t} \omega_{0}+\frac{\omega_{0}}{C_{g}\left(s^{2}+\omega_{0}^{2}\right)} \\ L_{g t} \omega_{0}-\frac{\omega_{0}}{C_{g}\left(s^{2}+\omega_{0}^{2}\right)} & R_{g}+L_{g t} s+\frac{C_{g}\left(s^{2}+\omega_{0}^{2}\right)}{C^{2}}\end{array}\right]$

If the grid voltage is neglected, as it does not have an influence on the system small signal stability, the grid current, $I_{g d q}$, is determined by the $L C L$ filter capacitor voltage

$$
V_{C f d q}=\left[Z_{g}\right]_{\omega_{0}} I_{g d q} .
$$

4) Plant Model: The combination of (7), (8), (9), (11), (12), (14) with

$$
I_{G d q}=I_{S d q}+I_{C f d q}+I_{g d q},
$$

allows to compute the complete plant model that correlates the four voltage inputs, $V_{G d q}$ and $V_{R d q}$, with the six model outputs $I_{G d q}, I_{R d q}$ and $V_{C f d q}$

$$
\left[\begin{array}{c}
I_{G d q} \\
I_{R d q} \\
V_{C f d q}
\end{array}\right]=[\text { Plant }]\left[\begin{array}{c}
V_{G d q} \\
V_{R d q}
\end{array}\right]
$$

Where $[$ Plant $]$ is a $6 \times 4$ transfer function matrix. Note that in this model, the influence and interaction between the RSC and the GSC variables is considered.

\section{B. RSC and GSC Control Loops}

As represented in Fig. 3, both the GSC and RSC are controlled as current sources in $d q$ axis by means of a PI controller. The elements that model the current control loops are represented in blue, while the PLL model is represented in red.

1) Current Control Loop Model: The GSC and RSC output currents are the variables controlled in the current control loops. As represented in Fig. 3, these variables are filtered by a low-pass analog filter, $L P A F=1 /(\tau s+1)$, to reduce the switching ripple. However, this filter is applied to the GSC currents in the stator stationary reference frame, $\alpha \beta_{S}$, and to the RSC currents in a different stationary reference frame that rotates at $\omega_{m}, \alpha \beta_{m}$.

To properly analyze the system stability, all the elements must be referred to the same reference frame, the $d q$ axis in this paper. For this reason, the transformation proposed in [13] to rotate the transfer functions between the different frames is used. The model of $L P A F$ in $d q$ axis, rotating at $\omega$, is a $2 \times 2$ transfer function matrix

$$
[L P A F]_{\omega}=\frac{1}{2}\left[\begin{array}{cc}
L P A F_{1}(s) & L P A F_{2}(s) \\
-L P A F_{2}(s) & L P A F_{1}(s)
\end{array}\right]
$$

whose diagonal term $\operatorname{LPAF}_{1}(s)=\operatorname{LPAF}(s+j \omega)+$ $\operatorname{LPAF}(s-j \omega)$ and anti-diagonal term $L P A F_{2}(s)=$ $j L P A F(s+j \omega)-j L P A F(s-j \omega)$. The low-pass analog filter in the GSC and the RSC have the same time constant, $\tau$, but each of them is defined in a different stationary reference frame. For this reason, for the GSC filter, the rotation is made with $\omega_{0}$, leading to the transfer function $[L P A F]_{\omega_{0}}$, while for the RSC, as the filter is defined in $\alpha \beta_{m}$ rotating at $\omega_{m}$, the transformation is made with $\omega_{R}=\omega_{0}-\omega_{m}$, leading to $[L P A F]_{\omega_{R}}$.

A PI controller is applied to the current error. The PI controller in the $d q$ axis is a diagonal matrix

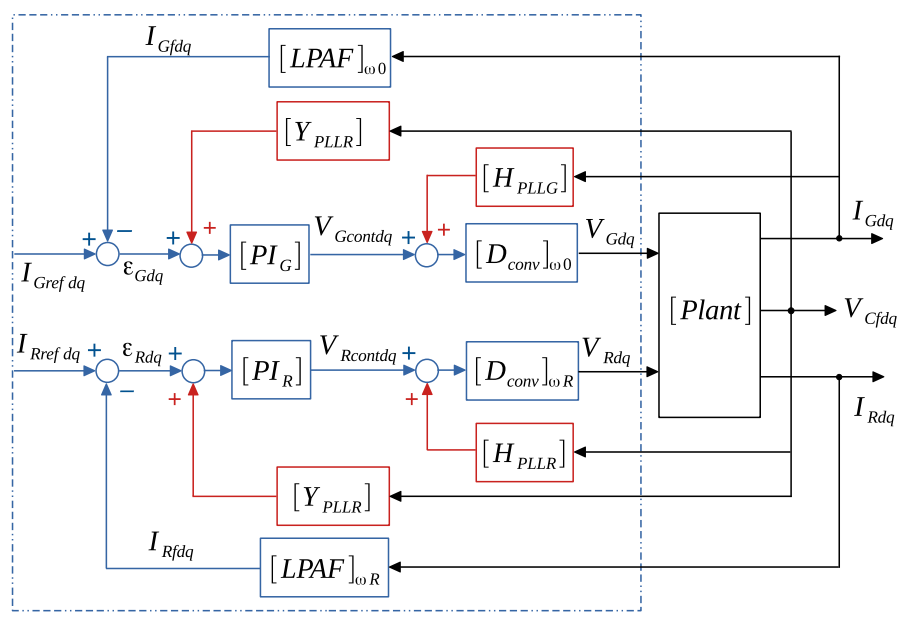

Fig. 3. GSC and RSC current control loops including the PLL model. 


$$
[P I]=\left[\begin{array}{cc}
K_{p} \frac{T_{n} s+1}{T_{n} s} & 0 \\
0 & K_{p} \frac{T_{n} s+1}{T_{n} s}
\end{array}\right] .
$$

The parameters for the RSC controller, $\left[P I_{R}\right]$, are different than the ones for the GSC, $\left[P I_{G}\right]$, as each current control loop has different dynamics.

The last element in the current control loop, $D_{\text {conv }}$, models the delays introduced by the power converter, i.e., the computation delay in the DSP and the zero order hold that represents the PWM power converter. A forth order approximation is used for $D_{c o n v}$, in order to achieve an accurate representation [14]. Similarly to the $L P A F, D_{\text {conv }}$ is transformed to $d q$ axis, leading to two different transfer function matrices; $\left[D_{\text {conv }}\right]_{\omega_{0}}$ for the GSC and $\left[D_{c o n v}\right]_{\omega_{R}}$ for the RSC.

2) PLL Model: The power converters are synchronized with the grid by means of a phase-locked loop. The PLL provides the angle for the transformation to the synchronous reference frame. Ideally, the grid voltage, $v_{g}$, would be measured, however, this is not a feasible solution, and normally the filter capacitor voltage is used instead, $v_{C f}$. The synchronization with $v_{C f}$ introduces an interaction between the PLL and the current control loop that can have an influence on the system stability at low frequencies [12], [15]. As SSRs occur at low frequencies, the PLL model is included in the stability analysis.

As shown in Fig. 4, the capacitor voltage in the stator stationary reference frame is filtered by a SOGI filter and transformed to $d q$ axis using the Park transformation $\left[P_{T}\right]$. The capacitor voltage $q$-component is driven to zero by a PI controller. The angular speed at the output of the PI controller is integrated to compute the angle and used in the feedback path for the Park transformation. The $[S O G I]$ filter in Fig. 4 is a diagonal matrix with diagonal terms $F S O G I=k \omega s /\left(s^{2}+k \omega s+\omega^{2}\right)$, that must be transformed to $d q$ coordinates using the previously mentioned transformation for $D_{\text {conv }}$ and $L P A F$.

The model for the PLL proposed in [12] represents the Park direct and inverse transformation as a disturbance, depicted in red in Fig. 3. The Park direct transformation introduces a disturbance dependent on the capacitor voltage in the RSC current control loop, $\left[Y_{P L L R}\right]$, and in the GSC one, $\left[Y_{P L L G}\right]$. Similarly, the inverse park transformation introduces a disturbance in the RSC, $\left[H_{P L L R}\right]$, and in the GSC, $\left[H_{P L L G}\right]$. These matrices are equal to

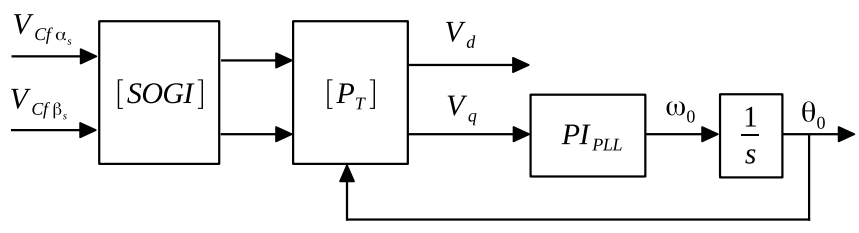

Fig. 4. Phase-locked loop used for grid synchronization.

$$
\left[H_{P L L}\right]=\left[\begin{array}{cc}
0 & -F T_{P L L} \overline{V_{q}} \\
0 & F T_{P L L} \overline{V_{d}}
\end{array}\right],
$$

and

$$
\left[Y_{P L L}\right]=\left[\begin{array}{cc}
0 & -F T_{P L L} \overline{I_{q}} \\
0 & F T_{P L L} \overline{I_{d}}
\end{array}\right]
$$

where

$$
F T_{P L L} \approx \frac{P I_{P L L} F S O G I_{1}}{s+P I_{P L L} F S O G I_{1} \overline{V_{C f d}}} .
$$

It should be noted that the matrices $\left[Y_{P L L}\right]$ and $\left[H_{P L L}\right]$ are different for the GSC and the RSC, as the steady-state currents, $\overline{I_{d}}$ and $\overline{I_{q}}$ in (20), equal $\overline{I_{G d q}}$ for the GSC and $\overline{I_{R d q}}$ for the RSC. Similarly, the voltages imposed by each control loop, $\overline{V_{d}}$ and $\overline{V_{q}}$ in (19), are different for the GSC, $\overline{V_{G d q}}$, and for the RSC, $\overline{V_{R d q}}$. At last, $\overline{V_{C f d}}$ is the filter capacitor voltage in the $d$-axis, as the $q$-component is driven to zero by the PLL,

\begin{tabular}{|c|c|}
\hline Parameter & Magnitude \\
\hline \multicolumn{2}{|c|}{$\begin{array}{c}\text { Distribution grid (Zorillo-Rio Hondo) } \\
S_{B}=100 M V A, U=345 \mathrm{kV}\end{array}$} \\
\hline $\begin{array}{l}\text { Line reactance } \\
\text { Line resistance }\end{array}$ & $\begin{array}{l}0.034 \text { p.u. } \\
0.003 \text { p.u. }\end{array}$ \\
\hline \multicolumn{2}{|c|}{$\begin{array}{c}\text { Wind farm substation } \\
S_{B}=250 \mathrm{MVA}, 34.5 / 345 \mathrm{kV}\end{array}$} \\
\hline $\begin{array}{l}\text { Reactance } \\
\text { Resistance }\end{array}$ & $\begin{array}{l}0.1 \text { p.u. } \\
0.004 \text { p.u. }\end{array}$ \\
\hline \multicolumn{2}{|c|}{$\begin{array}{c}\text { Wind farm grid } \\
S_{B}=100 M V A, U=34.5 \mathrm{kV}\end{array}$} \\
\hline $\begin{array}{l}\text { Line reactance } \\
\text { Line resistance }\end{array}$ & $\begin{array}{l}0.013 \text { p.u. } \\
0.008 \text { p.u. }\end{array}$ \\
\hline \multicolumn{2}{|c|}{$\begin{array}{c}\text { Wind turbine transformer } \\
S_{B}=2.5 \mathrm{MVA}, 34.5 / 0.69 \mathrm{kV}\end{array}$} \\
\hline $\begin{array}{l}\text { Reactance } \\
\text { Resistance }\end{array}$ & $\begin{array}{l}0.05 \text { p.u. } \\
0.007 \text { p.u. }\end{array}$ \\
\hline \multicolumn{2}{|c|}{$\begin{array}{c}\text { DFIG } \\
S_{B}=2.5 M V A, U=690 \mathrm{~V}\end{array}$} \\
\hline $\begin{array}{l}\text { Number of turbines } \\
\text { Rotor resistance } \\
\text { Rotor leakage inductance } \\
\text { Stator resistance } \\
\text { Stator leakage inductance } \\
\text { Magnetizing inductance }\end{array}$ & $\begin{array}{l}100 \\
0.008 \text { p.u. } \\
0.102 \text { p.u. } \\
0.006 \text { p.u. } \\
0.097 \text { p.u. } \\
4.34 \text { p.u. }\end{array}$ \\
\hline \multicolumn{2}{|c|}{ Power converter } \\
\hline Rated power & $500 \mathrm{kVA}$ \\
\hline Switching frequency & $2.8 \mathrm{kHz}$ \\
\hline Sampling frequency & $5.6 \mathrm{kHz}$ \\
\hline GSC reactance & 0.2 p.u. \\
\hline GSC resistance & 0.005 p.u. \\
\hline GSC filter capacitor & 0.036 p.u. \\
\hline GSC filter series damping resistance & 0.21 p.u. \\
\hline \multicolumn{2}{|c|}{ Control parameters } \\
\hline GSC PI proportional gain & 0.15 \\
\hline GSC PI integral constant & 0.03 \\
\hline RSC PI proportional gain & 0.14 \\
\hline RSC PI integral constant & 0.016 \\
\hline Low-pass analog filter time constant & $20.10^{-6}$ \\
\hline PLL PI proportional gain & 0.2 \\
\hline PLL PI integral constant & 0.22 \\
\hline SOGI proportional gain & 0.2 \\
\hline
\end{tabular}
so $F T_{P L L}$ is the same for both converters.

TABLE I

SYSTEM PARAMETERS 


\section{Stability Analysis}

A preliminary stability analysis is performed for the system parameters summarized in Table I. These are the parameters of a real SSR event reported in the southern Electric Reliability Council of Texas (ERCOT) [2]. 100 wind turbines are connected at the PCC, and thus, an aggregated model is used to analyze the stability [16].

The stability of any MIMO system, as the $2 \times 2$ dynamic model obtained for the current control loop of a DFIG wind turbine controlled in the synchronous reference frame, can be analyzed through the Bode diagram of the open-loop matrix eigenvalues [11]. According to this criterion (MIMO GBC), the number of closed-loop unstable poles, $Z$, is equal to the number of open-loop unstable poles, $P$, minus the total number of $\pm m 180$ degrees crossings ( $m$ odd integer) with positive magnitude counted in the Bode diagram of all the system open-loop eigenvalues, $C^{+}$(crossings with increasing phase), $C^{-}$(with decreasing phase) and $C_{0}$ (at $0 \mathrm{~Hz}$ )

$$
Z=P-\left[2\left(C^{+}-C^{-}\right)+C_{0}\right] .
$$

Fig. 5 (a) shows the two eigenvalues of the RSC current control loop, which is a $2 \times 2$ MIMO model, for two different slips $=\omega_{R} / \omega_{0}$, when the line series capacitor, $C_{g}$, compensates an $80 \%$ of the line impedance. The open-loop transfer function matrix correlates $I_{R d q}$ to $\varepsilon_{R d q}$ in Fig. 3. Note that in the stability analysis, even though only the RSC current control loop eigenvalues are represented, the open-loop matrix for the RSC includes the influence of the GSC current control loop, forcing $I_{\text {Gref } d q}=0$. For the two rotational speeds, the set of eigenvalues has a negative crossing at $37 \mathrm{~Hz}\left(C^{-}=1\right)$, so as the system has no open-loop unstable poles $(P=0)$, according to (22) the closed-loop system has 2 unstable poles for each slip, $Z=2$. If the line impedance compensation level is reduced to $50 \%$, Fig. 5 (b), the same number of crossings occur at $42 \mathrm{~Hz}$ for the two sets of eigenvalues, and thus, the closed-loop system has two unstable poles for each rotational speed.

It becomes evident from the stability analysis that a subsynchronous resonance damping (SSRD) strategy is required. The next section presents the proposed control strategy.

\section{SSR DAMPER}

The sub-synchronous instability identified in the previous section is the result of the interaction between the DFIG, the current control loops and the grid. Intuitively, from the analysis of Fig. 1, if the RSC of each wind turbine can perform a perfect feed-forward of the output $L C L$ filter voltage, $v_{C f}$, the DFIG wind turbine is immune to SSRs, as the grid impedance influence on the plant to be controlled is eliminated [17].

The sub-synchronous resonance damper proposed in this paper is based on the stator voltage, which is equal to the $L C L$ filter capacitor voltage, a variable that is already measured in DFIG wind turbines for grid synchronization, as previously described. The SSRD strategy is a positive feedback loop represented in Fig. 6 in green. The filter capacitor voltage (or equivalently the stator voltage), filtered by a low-pass analog filter and multiplied by the SSR damping controller, $\left[F F_{R}\right]$, provides the voltage action, $V_{R D d q}$, to be applied in the RSC. The goal of $\left[F F_{R}\right]$ is to compensate the grid impedance and voltage.

As the DFIG wind turbine is controlled from the RSC, the stator voltage has to be referred to the rotor terminals. To do so, the previously proposed model of a DFIG wind turbine is converted to a block diagram and represented in Fig. 7, neglecting the winding series resistance. It becomes clear that there are two inputs to the model: the rotor voltage applied by the RSC, $V_{R d q}$, and the stator voltage, $V_{S d q}$. In order to compensate the stator voltage with the action applied by the rotor-side converter, $\left[F F_{R}\right]$ has to equal

$$
\left[F F_{R}\right]=[B]^{-1}\left(I+\left[Z_{L_{l R}}\right]_{\omega_{0}}\left[Z_{L_{m}}\right]_{\omega_{0}}^{-1}\right),
$$

where $I$ is the order two identity matrix.

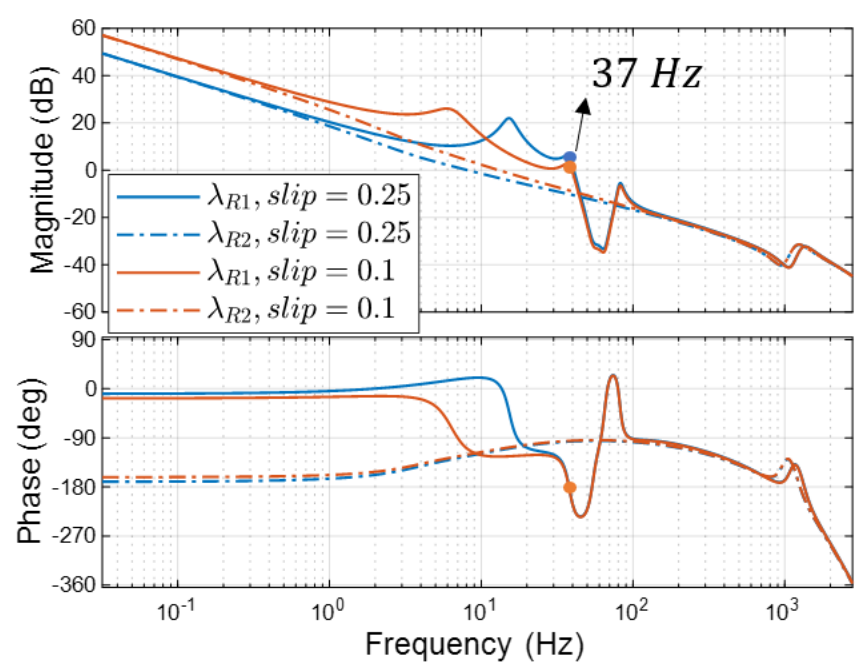

(a)

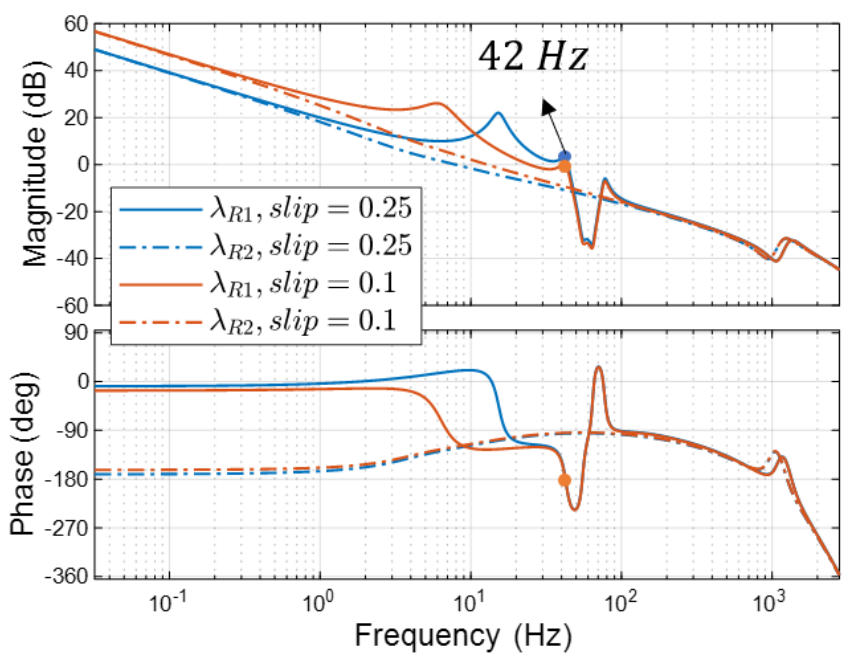

(b)

Fig. 5. Eigenvalues' Bode diagrams of the RSC current control loop for two slips when the line impedance compensation level is $80 \%$ (a) and $50 \%$ (b). 
However, as indicated by the control diagram in Fig. 6, the GSC and RSC interact with each other as they are two power converters connected in parallel to the grid through the DFIG and $L_{G S C}$, respectively. This interaction must be considered in the design procedure of the SSRD, as the stability of both power converters is strongly influenced by each other and the SSRD cannot be adjusted just with the expression in (23).

In Fig. 8 (a) the eigenvalues of the RSC inner SSR damper loop, correlating $V_{R D d q}$ with $V_{R \text { contdq }}$ are represented for $\left[F F_{R}\right]$ equal to (23) (blue curves), considering the GSC current control loop influence (with $I_{\text {Gref } d q}=0$ ). The slip is 0.25 and the series compensation capacitor, $C_{g}$, compensates $80 \%$ of the line impedance. Only the lowest rotational speed is represented, because it is the case that introduces a greater instability, given that the magnitude in the Bode diagrams of Fig. 5 is higher at reduced DFIG rotational speeds for both line impedance compensation levels. These results match the ones published by [1]. According to the MIMO GBC, as there are two $\pm m 180$ degree ( $m$ odd integer) crossings with positive magnitude, marked by the blue dots, the capacitor voltage positive feedback introduces 4 unstable poles. Similarly, in Fig. 8 (b), for a slip $=0.25$ and a $50 \%$ line impedance compensation (blue curves), the same conclusion is drawn. In both cases, $\lambda_{F F R 1}$ has two negative crossings, and thus, the stator voltage positive feedback loop introduces four unstable poles.

Nevertheless, the SSR instability is only introduced in one of the eigenvalues at positive frequencies, $\lambda_{F F R 1}$. Consequently, if a rotation is introduced in the stator voltage feedback without changing the magnitude plot, these $\pm m 180$ degree crossings can be avoided. (24) shows the proposed subsynchronous resonance damper when a rotation matrix $[R M]$, by an angle $\alpha$, is applied

$$
\begin{aligned}
{\left[F F_{R}\right] } & =[R M][B]^{-1}\left(I+\left[Z_{L_{l R}}\right]_{\omega_{0}}\left[Z_{L_{m}}\right]_{\omega_{0}}^{-1}\right) \\
& =\left[\begin{array}{cc}
\cos \alpha & -\sin \alpha \\
\sin \alpha & \cos \alpha
\end{array}\right] \times\left[\begin{array}{cc}
\frac{L_{R}}{L_{m}} \frac{s^{2}+\omega_{0} \omega_{R}}{s^{2}+\omega_{0}^{2}} & \frac{L_{R}}{L_{m}} \frac{s \omega_{m}}{s^{2}+\omega_{0}^{2}} \\
-\frac{L_{R}}{L_{m}} \frac{s \omega_{m}}{s^{2}+\omega_{0}^{2}} & \frac{L_{R}}{L_{m}} \frac{s^{2}+\omega_{0} \omega_{R}}{s^{2}+\omega_{0}^{2}}
\end{array}\right],
\end{aligned}
$$

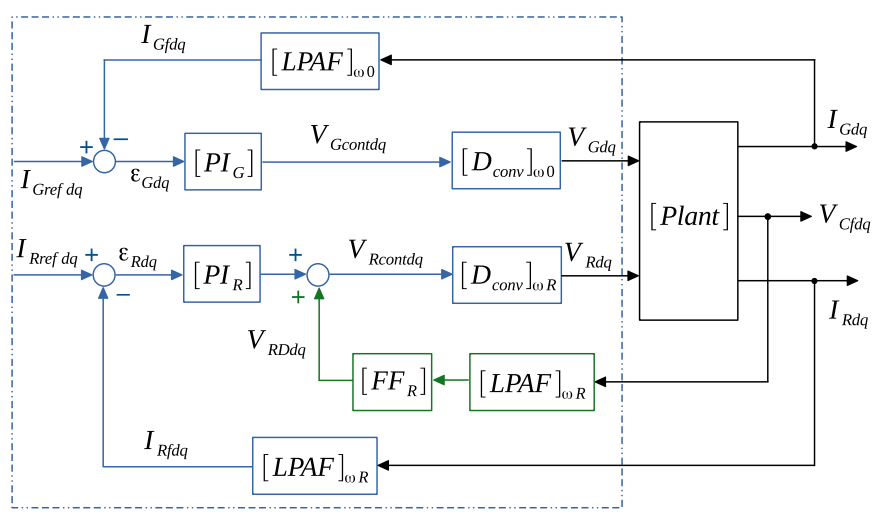

Fig. 6. GSC and RSC current control loops with the SSRD represented in green.

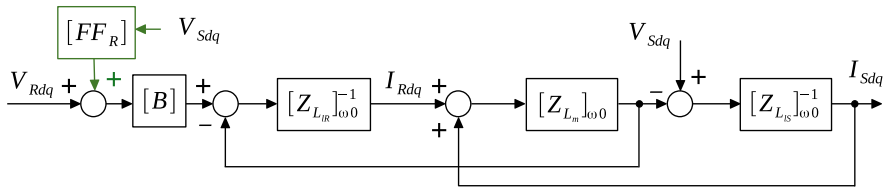

Fig. 7. Block diagram representation of the DFIG matrix impedance model.

where $L_{R}$ is the sum of $L_{m}$ and the rotor leakage inductance, $L_{l R}$.

A -120 degree rotation is added $(\alpha=-120)$, and the eigenvalues are represented again (orange curves in Fig. 8 (a) and (b)). With this rotation, the SSRD does not introduce any unstable poles, as the resulting eigenvalues do not present any crossing with positive magnitude. Thus, the stabilizing effect in the outer current control loop of the proposed controller based on the $\left[F F_{R}\right]$ matrix in (24) can be evaluated.

The open-loop matrix eigenvalues of the RSC and the GSC current control loops, that correlate $I_{R f d q}$ with $\varepsilon_{R d q}$ (blue

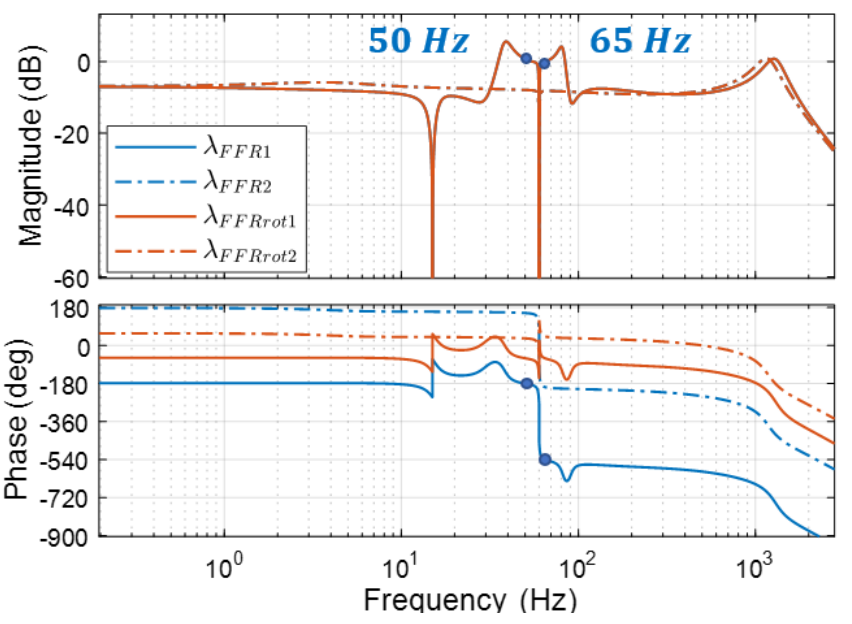

(a)
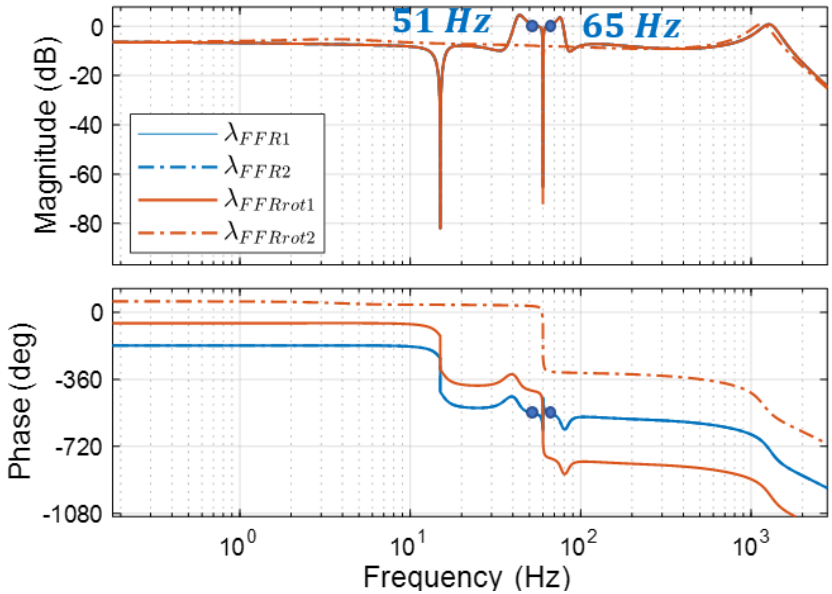

(b)

Fig. 8. Inner SSR damper loop eigenvalues' Bode diagram without a rotation in $\left[F F_{R}\right]$ (blue) and for a -120 degree rotation (orange) with an $80 \%$ line impedance compensation (a) and 50\% (b). 

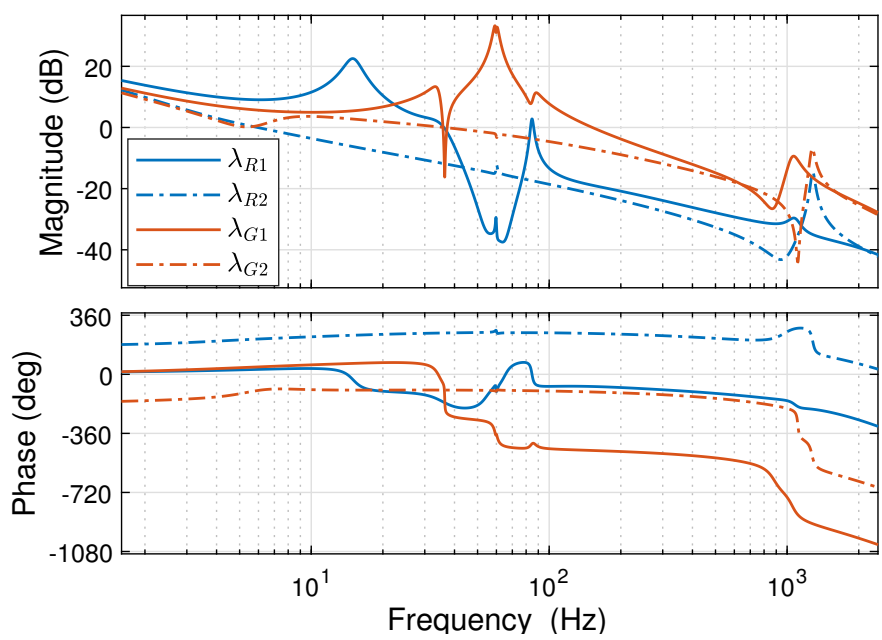

(a)
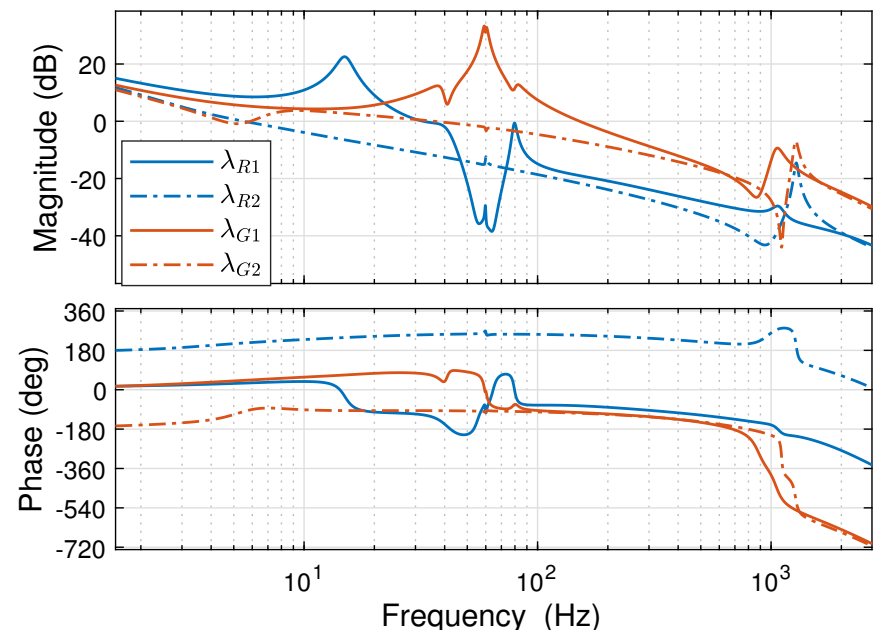

(b)

Fig. 9. RSC (blue) and GSC (orange) current control loop eigenvalues' Bode diagram with the proposed SSRD and an $80 \%$ line impedance compensation (a) and $50 \%$ (b).

curves), and $I_{G f d q}$ with $\varepsilon_{G d q}$ (orange curves), are represented in Fig. 9 for a slip equal to 0.25 . These open-loop transfer function matrices include the inner SSRD adjusted previously. It can be seen that neither the RSC nor the GSC eigenvalues have $\pm m 180$ degree crossings with positive magnitude, and thus the closed-loop current control loops are stable.

The analysis performed through the eigenvalues' Bode diagram and the MIMO GBC is confirmed by the closedloop pole representation in Fig. 10. The closed-loop poles are represented for all the range of possible rotational speeds (wind speeds), and three line impedance compensation levels $80 \%, 50 \%$ and $20 \%$. In all the cases, the system is stable, proving that the SSR damper is effective and robust.

\section{Simulation REsults}

To validate the proposed sub-synchronous resonance damper, a model is created in Matlab using the Simscape Elec-

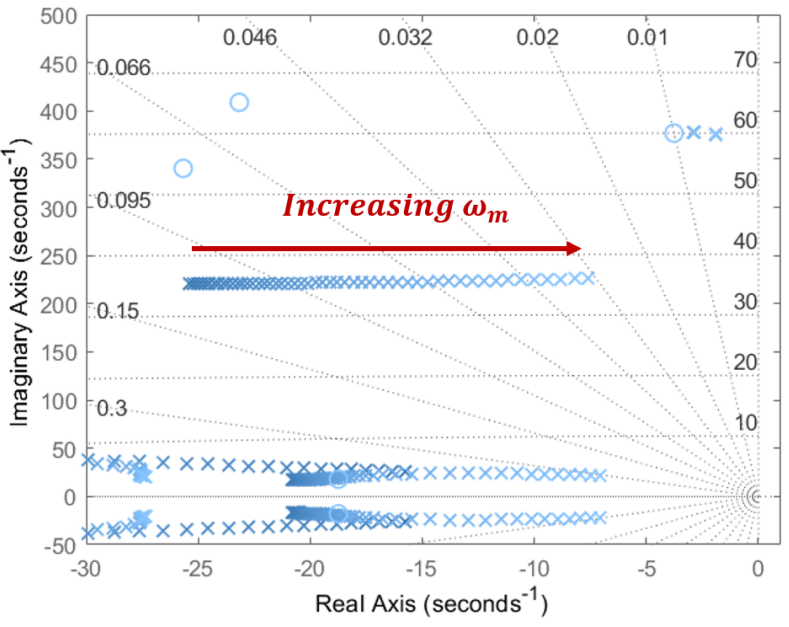

(a)

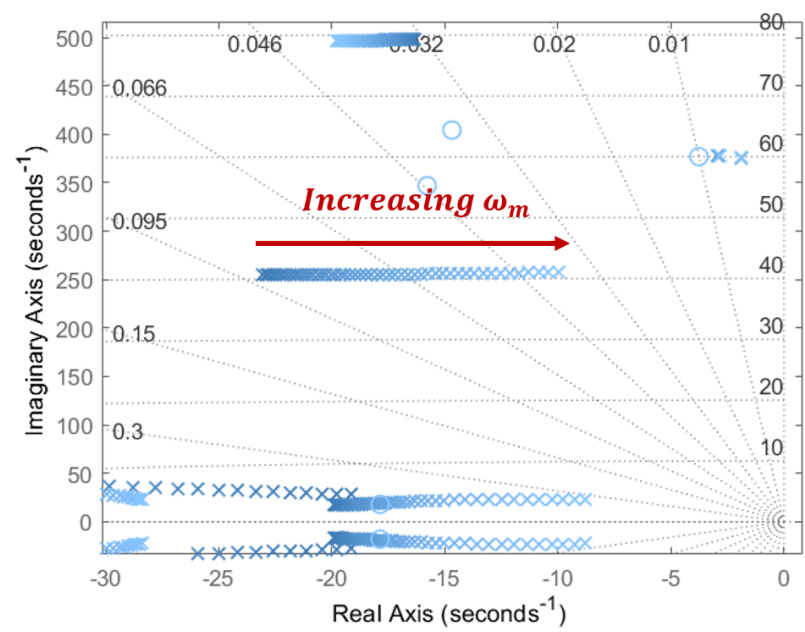

(b)

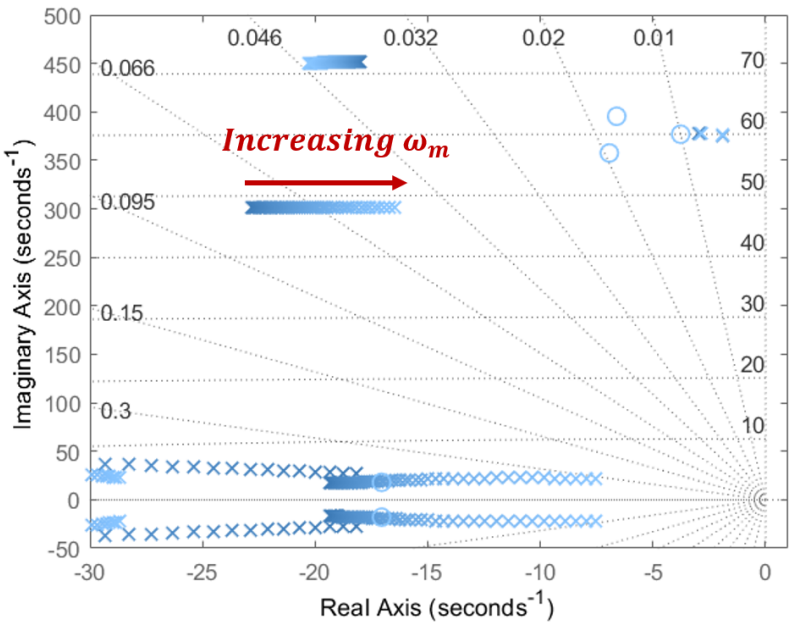

(c)

Fig. 10. Closed-loop pole evolution for the whole range of possible rotational speeds for an $80 \%$ line impedance compensation (a), $50 \%$ (b), and $20 \%$ (c).

trical Library. The system parameters are the ones specified in Table I.

Fig. 11 shows the grid current evolution as the proposed 


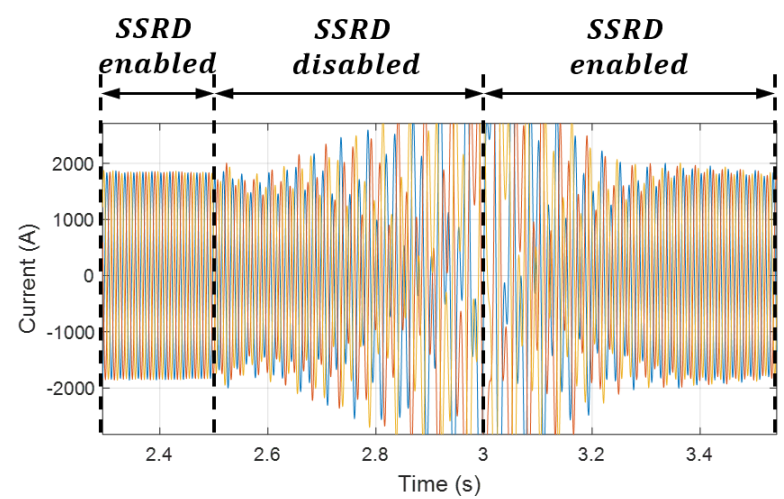

(a)

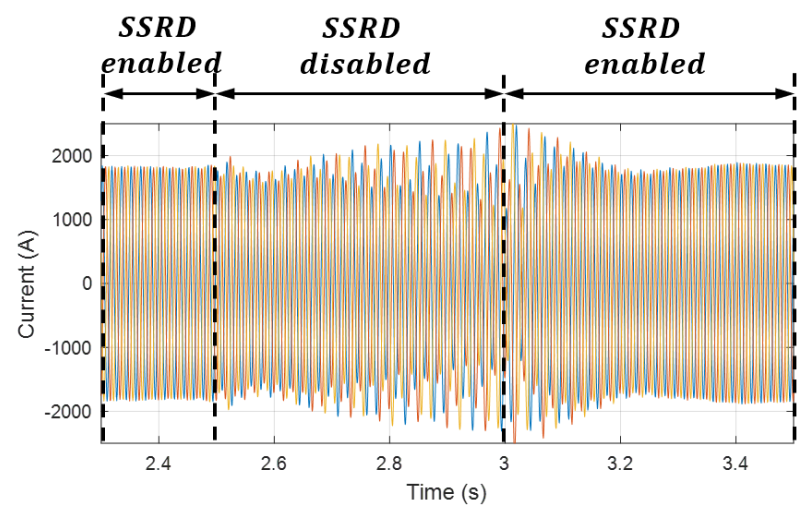

(b)

Fig. 11. Grid current transient simulation disabling the SSR damping strategy at $2.5 \mathrm{~s}$ for an $80 \%$ (a) and a $50 \%$ (b) line impedance compensation level.

SSRD is transiently disabled. At the beginning of the simulation, the system is stable, but when the SSRD is disabled $\left(\left[F F_{R}\right]=[0]\right)$ at $2.5 \mathrm{~s}$, the grid current becomes unstable and a SSR component appears. However, if the damping control strategy is enabled at $3 \mathrm{~s}$ the system becomes stable again. The simulation is performed for an $80 \%$ and a $50 \%$ line impedance compensation level and with a slip $=0.25$, the minimum rotational speed. These results confirm the stability analysis and the conclusions drawn from Fig. 5 and Fig. 9. It also proves the robustness of the proposed SSRD.

\section{CONCLUSiON}

In this paper a sub-synchronous resonance damper implemented in the RSC and based on the capacitor voltage positive feedback is proposed. The damping strategy depends on the DFIG impedances and a rotation. The DFIG impedances are used to refer the stator voltage to the rotor terminals, where the RSC is connected. In contrast, the rotation guarantees the stability regardless of the DFIG operation point and the interaction of the RSC with the GSC. The proposed control strategy is robust, as it can stabilize the system for any line impedance compensation level and DFIG rotational speed, meaning that it is stable for any wind speed. Moreover, the sub-synchornous resonance damper is based on local and already measured variables in DFIG wind turbines, i.e. the output filter capacitor voltage, so it can be implemented in any new or existing DFIG wind turbine with minor changes in the control algorithm.

\section{REFERENCES}

[1] L. Fan, R. Kavasseri, Z. L. Miao, and C. Zhu, "Modeling of dfig-based wind farms for ssr analysis," IEEE Transactions on Power Delivery, vol. 25, no. 4, pp. 2073-2082, 2010.

[2] A. E. Leon and J. A. Solsona, "Sub-synchronous interaction damping control for dfig wind turbines," IEEE Transactions on Power Systems, vol. 30, no. 1, pp. 419-428, 2015.

[3] R. K. Varma, S. Auddy, and Y. Semsedini, "Mitigation of subsynchronous resonance in a series-compensated wind farm using facts controllers," IEEE Transactions on Power Delivery, vol. 23, no. 3, pp. $1645-1654,2008$.

[4] L. Fan and Z. Miao, "Mitigating ssr using dfig-based wind generation," IEEE Transactions on Sustainable Energy, vol. 3, no. 3, pp. 349-358, 2012.

[5] H. A. Mohammadpour and E. Santi, "Ssr damping controller design and optimal placement in rotor-side and grid-side converters of seriescompensated dfig-based wind farm," IEEE Transactions on Sustainable Energy, vol. 6, no. 2, pp. 388-399, 2015.

[6] A. E. Leon, "Integration of dfig-based wind farms into seriescompensated transmission systems," IEEE Transactions on Sustainable Energy, vol. 7, no. 2, pp. 451-460, 2015.

[7] H. J. Baesmat and M. Bodson, "Suppression of sub-synchronous resonances through excitation control of doubly fed induction generators," IEEE Transactions on Power Systems, vol. 34, no. 6, pp. 4329-4340, 2019.

[8] X. Zhang, Y. Zhang, R. Fang, and D. Xu, "Impedance modeling and ssr analysis of dfig using complex vector theory," IEEE Access, vol. 7, pp. $155860-155870,2019$.

[9] P. Huang, M. S. El Moursi, W. Xiao, and J. L. Kirtley, "Subsynchronous resonance mitigation for series-compensated dfig-based wind farm by using two-degree-of-freedom control strategy," IEEE Transactions on Power Systems, vol. 30, no. 3, pp. 1442-1454, 2015.

[10] H. Liu, X. Xie, Y. Li, H. Liu, and Y. Hu, "Mitigation of ssr by embedding subsynchronous notch filters into dfig converter controllers," IET Generation, Transmission \& Distribution, vol. 11, no. 11, pp. 28882896, 2017.

[11] J. Samanes, A. Urtasun, E. L. Barrios, D. Lumbreras, J. Lopez, E. Gubia, and P. Sanchis, "Control design and stability analysis of power converters: The mimo generalized bode criterion," IEEE Journal of Emerging and Selected Topics in Power Electronics, vol. 8, no. 2, pp. 1880-1893, 2020.

[12] B. Wen, D. Boroyevich, R. Burgos, P. Mattavelli, and Z. Shen, "Analysis of d-q small-signal impedance of grid-tied inverters," IEEE Transactions on Power Electronics, vol. 31, no. 1, pp. 675-687, 2016.

[13] D. N. Zmood, D. G. Holmes, and G. H. Bode, "Frequency-domain analysis of three-phase linear current regulators," IEEE Transactions on Industry Applications, vol. 37, no. 2, pp. 601-610, 2001.

[14] J. Samanes, A. Urtasun, E. Gubia, and A. Petri, "Robust multisampled capacitor voltage active damping for grid-connected power converters," International Journal of Electrical Power \& Energy Systems, vol. 105, pp. 741-752, 2019.

[15] X. Wang, L. Harnefors, and F. Blaabjerg, "Unified impedance model of grid-connected voltage-source converters," IEEE Transactions on Power Electronics, vol. 33, no. 2, pp. 1775-1787, 2018.

[16] J. L. Agorreta, M. Borrega, J. López, and L. Marroyo, "Modeling and control of $n$-paralleled grid-connected inverters with lcl filter coupled due to grid impedance in pv plants," IEEE Transactions on Power Electronics, vol. 26, no. 3, pp. 770-785, 2010.

[17] J. Samanes, E. Gubia, and J. Lopez, "Mimo based decoupling strategy for grid connected power converters controlled in the synchronous reference frame," in 2018 IEEE 19th Workshop on Control and Modeling for Power Electronics (COMPEL), 2018, pp. 1-8. 